\title{
Role of GeneXpert MTB/Rif in detection of tuberculosis from clinical samples: A report from a tertiary care hospital in Mumbai
}

\author{
Sunayana M. Jangla ${ }^{1, *}$, Susan Cherian ${ }^{2}$, Raji Naidu ${ }^{3}$ \\ ${ }^{1}$ Microbiologist, ${ }^{2}$ Pathologist \& HOD, ${ }^{3}$ Pathologist, Dept. of Pathology, Bhabha Atomic Research Centre (BARC) Hospital, \\ Anushakti Nagar, Trombay, Mumbai, Maharashtra, India
}

*Corresponding Author:

Email: sunayanajangla79@gmail.com

\begin{abstract}
Introduction: Tuberculosis (TB) causes severe morbidity and mortality in millions of people every year, worldwide and India in particular. Hence this study was undertaken to find out the role of the widely used GeneXpertMTB/Rif test in tuberculosis detection.

Materials and Methods: A total of 155 samples (pulmonary and extrapulmonary) from patients clinically suspected to have tuberculosis were received for testing by ZN (Ziehl-Neelsen) stain and GeneXpert/MTB Rif. These samples were also processed for histopathology or cytology and mycobacterial culture on request of clinician. The results were studied and compared.

Result: Out of the total samples received, lymph node aspirates were $23 \%$,pleural fluid $20 \%$,sputum $19 \%$,pus $10 \%$,tissue $8 \%$,ascitic fluid $8 \%$,cerebrospinal fluid $7 \%$,endometrial tissue $3 \%$,synovial fluid $1 \%$ and urine $1 \%$.Nine sputum samples $(31 \%)$ were positive by GeneXpertMTB/Rif of which two were negative on smear, two positive on culture and there was no culture request for the remaining seven samples.29 (23\%) extrapulmonary samples were positive by GeneXpertMTB/Rif out of which there was no culture request for 16 samples. One sample which was negative by GeneXpert MTB/Rif grew Atypical Mycobacteria on culture. Four isolates were detected as rifampicin resistant by GeneXpertMTB/Rif but none of them were sent for Drug Susceptibility Test (DST).
\end{abstract}

Conclusion: GeneXpertMTB/Rif should be used in combination with other tests for diagnosing tuberculosis.

Keywords: Drug resistant tuberculosis, GeneXpert/MTB Rif, Nucleic-acid amplification test.

\section{Introduction}

Dr. Robert Koch isolated Mycobacterium tuberculosis, the microorganism responsible for Tuberculosis in 1882. ${ }^{1}$ Since then, efforts are being made worldwide to diagnose and treat this deadly disease. India has a very high burden of $\mathrm{TB}$ in the world. ${ }^{2}$ According to World Health Organisation (WHO) report in 2015, 10.4 million new TB cases were found worldwide and 1.8 million TB deaths. India was one of those countries where most of these new cases were found. ${ }^{2}$ Not only is the burden of pulmonary tuberculosis very high but extrapulmonary tuberculosis also accounts for approximately $25 \%$ all TB cases. ${ }^{3}$ Hence accurate and timely diagnosis not only helps in starting the treatment early but also helps in preventing development of resistance and decreasing the risk of spread of this bug in the community. Multidrug Resistant Tuberculosis (MDR-TB) is difficult and expensive to treat. Till date, detection of Mycobacteria remains a challenge. There are different ways of diagnosing it in the laboratory. Conventional methods are microscopic detection using different stains(smear microscopy),histopathology of the affected tissue, Fine Needle Aspiration Cytology (FNAC )of the involved organ and culture using solid media (Lowenstein Jensen).Some newer techniques include culture by automated systems (e.g. BacT/Alert 3D,MGIT 960) using liquid media, immunological methods like TB Gold etc, and molecular methods like GeneXpertMTB/Rif assay (Cephid Sunnyvale,
California, United States) and Heinz test (PCR Line Probe Assay). ${ }^{4}$ Each method has its own advantages and drawbacks depending on the time taken, cost involved and availability. GeneXpert MTB/Rif assay is a hemi-nested Polymerase Chain Reaction (PCR) which simultaneously detects DNA of Mycobacterium tuberculosis complex (MTBC) and resistance to rifampicin (RIF) which is a surrogate marker for the identification of multi-drug resistant tuberculosis (MDR -TB) in less than 2 hours. Due to its decreased turnaround time, WHO recommended this assay in TB endemic countries for all individuals (smear-negative, paediatric, MDR-TB and Human Immunodeficiency Virus [HIV]) suspected of having pulmonary and extrapulmonary TB in October 2010.5

\section{Material and Methods}

The institute where this study was conducted is a 360 bedded multispeciality tertiary care hospital in Mumbai which provides health care facilities to its employees and their members. Here, detection of Mycobacteria is done using various methods like smear microscopy (Ziehl-Neelsen stain), GeneXpert MTB/ Rif and histopathology and cytology. Samples for Mycobacterium culture are currently outsourced in view of the infrastructure constraints. A total of 155 samples (pulmonary and extrapulmonary) were received for testing by GeneXpert MTB/Rif assay and smear microscopy during the period of January 2016 to June 2017 (18 months) from patients clinically suspected to 
have tuberculosis. Age and gender of the patients were noted. Also, it was noted whether these patients had past history of infection with TB or Anti Koch's Treatment (AKT) was taken and whether there was presence of immunocompromised conditions like HIV infection, malignancy etc. Smear microscopy was done by staining smears with Ziehl-Neelsen stain according to standard recommended procedure ${ }^{4}$ and examined microscopically. GeneXpert MTB/Rif assay was performed according to manufacturer's instructions (Cephid, Sunnyvale, California, United States). Culture samples were outsourced to a referral laboratory only on clinician's request where TB culture is done using MGIT 960 liquid culture (Becton Dickinson, Sparks, MD, USA) and Lowenstein-Jensen (solid) media. Samples for FNAC (lymph nodes aspirates) and histopathology (biopsy) were collected and processed in the respective section only on clinician's request.

\section{Results}

Out of the 155 samples received, various types of samples and their number is shown in Table 1. Male patients were $88(57 \%$ ) whereas females were $67(43 \%)$. $109(70 \%)$ samples were from age group 19-65 years, $40(26 \%)$ samples were from patients above 65 years and six samples from patients up to 18 years. Symptoms suggestive of tuberculosis were present in all patients with or without radiological findings. 18 patients had past history of either TB or had taken AKT. 15 patients suffered from Bronchial asthma and 13 patients had Alcoholic liver disease with/without cirrhosis. Patients with malignancy, chronic renal failure, primary infertility and HIV/AIDS were 13, 11, five and four respectively. Two patients had history of contact with infected person. Comparison of GeneXpert MTB/Rif assay results with other methods done on sputum samples and extrapulmonary samples are shown in Table 2 and Table 3 respectively. Out of nine (31\%) sputum samples which tested positive by GeneXpert MTB/Rif assay, seven samples were positive on smear microscopy while two were negative. Growth of Mycobacterium tuberculosis complex (MTBC) was seen in culture of two samples and no culture requisition was there for seven samples. 20 sputum samples tested negative for GeneXpert/MTB Rif assay of which all were negative by smear microscopy and culture of one sample did not show growth while there was no requisition of culture for remaining 19 samples. Of the remaining 126 extrapulmonary samples,29 tested positive by GeneXpert MTB/Rif assay of which 14 were positive and 15 were negative by smear microscopy, six samples grew $M T B C$, seven did not show growth and there was no requisition for culture in 16 samples. 22 were positive while three were negative for TB on histopathology or cytology. Out of the 97 samples that tested negative by GeneXpert MTB/Rif assay, one showed Acid-Fast Bacilli (AFB) on microscopy while 96 were negative for AFB. One sample grew Mycobacterium fortuitum while 19 samples did not show growth and culture for remaining 77 was not requested and one sample was positive for TB by histopathology while 27 were negative. Four isolates were detected as Rifampicin resistant by GeneXpert.

Table 1: Types of samples received

\begin{tabular}{|l|c|}
\hline \multicolumn{1}{|c|}{ Samples } & Number (\%) \\
\hline Lymph node aspirate & $36(23)$ \\
\hline Pleural fluid & $31(20)$ \\
\hline Sputum & $29(19)$ \\
\hline Pus & $16(10)$ \\
\hline Tissue & $13(8)$ \\
\hline Ascitic fluid & $12(8)$ \\
\hline Cerebrospinal fluid & $11(7)$ \\
\hline Endometrial tissue & $04(3)$ \\
\hline Synovial fluid & $02(1)$ \\
\hline Urine & $01(1)$ \\
\hline Total & 155 \\
\hline
\end{tabular}

Table 2: Comparison of GeneXpert MTB/Rif assay results with other methods done on sputum samples

\begin{tabular}{|c|c|c|c|c|c|}
\hline $\begin{array}{c}\text { GeneXpert } \\
\text { MTB/Rif }\end{array}$ & \multicolumn{2}{|c|}{$\begin{array}{c}\text { Smear Microscopy } \\
\text { (ZN Stain) }\end{array}$} & \multicolumn{3}{c|}{ Culture } \\
\hline $\begin{array}{c}\text { Positive } \\
09\end{array}$ & Positive & Negative & Positive & Negative & $\begin{array}{c}\text { Not } \\
\text { requested }\end{array}$ \\
\cline { 2 - 6 } & 07 & 02 & 02 & 0 & 07 \\
\hline $\begin{array}{c}\text { Negative } \\
20\end{array}$ & Positive & Negative & Positive & Negative & $\begin{array}{c}\text { Not } \\
\text { requested }\end{array}$ \\
\cline { 2 - 6 } & 0 & 20 & 0 & 01 & 19 \\
\hline
\end{tabular}


Table 3: Comparison of GeneXpertMTB/Rif assay results with other methods done on extrapulmonary samples

\begin{tabular}{|c|c|c|c|c|c|c|c|}
\hline $\begin{array}{c}\text { GeneXpert } \\
\text { MTB/Rif }\end{array}$ & \multicolumn{2}{|c|}{$\begin{array}{l}\text { Smear Microscopy } \\
\text { (ZN Stain) }\end{array}$} & \multicolumn{3}{|c|}{ Culture } & \multicolumn{2}{|c|}{$\begin{array}{c}\text { Histopathology/Cytology } \\
\text { (tissue } \\
\text { biopsy/lymph node } \\
\text { aspirates) }\end{array}$} \\
\hline \multirow[t]{2}{*}{$\begin{array}{c}\text { Positive } \\
29\end{array}$} & Positive & Negative & Positive & Negative & $\begin{array}{c}\text { Not } \\
\text { requested }\end{array}$ & Positive & Negative \\
\hline & 14 & 15 & 06 & 07 & 16 & 22 & 03 \\
\hline \multirow[t]{2}{*}{$\begin{array}{l}\text { Negative } \\
97\end{array}$} & Positive & Negative & Positive & Negative & $\begin{array}{c}\text { Not } \\
\text { requested }\end{array}$ & Positive & Negative \\
\hline & 01 & 96 & 01 & 19 & 77 & 01 & 27 \\
\hline
\end{tabular}

\section{Discussion}

One of the most obvious and significant reason for using GeneXpert/MTB Rif is its decreased turn-around time along with easy technicality and less space requirement. In our institute, total of 38 samples were detected positive for $M T B C$ by GeneXpert MTB/Rif while microscopy detected AFB in 21 samples which shows that GeneXpert is more sensitive than smear microscopy. This was in accordance to a study by Munir KM. ${ }^{6}$ Various studies on performance of GeneXpert MTB/Rif showed that the test sensitivity for culture-positive/smear-negative cases ranges from 57\% to $76.9 \%$ while in culture-positive/smear-positive it ranges from $98 \%$ to $100 \% .^{7}$ A study by Rufai BS et al showed that its detection rate in smear-negative/culturepositive is low. ${ }^{8}$ Also, it can give false-negative and false-positive RIF resistance results and approximately $35 \%$ of their cases were detected as falsely RIF susceptible. ${ }^{8-11}$ Hence this might be a dangerous step for TB control and accentuate spread of MDR-TB. Rufai et al also found high discordance between culture and Xpert MTB/Rif results. ${ }^{8}$ In our case, out of the Rifampicin sensitive samples on GeneXpert, only three were sent for drug susceptibility testing (DST) of which one was sputum and all three were sensitive to rifampicin by culture also. However, the number of samples requested for culture and DST was very small in our case. Saeed $M$ et al found that GeneXpert detected false positive cases which were sensitive on Drug susceptibility testing (DST) possibly due to mixed infections where GeneXpert detects the strain which is predominately present. ${ }^{7}$ In our case, four samples were detected as Rifampicin resistant of which one was sputum and remaining three were extrapulmonary. Of these four samples, three grew MTBC but DST was not requested for any of them. Tortoli $\mathrm{E}$ et al found that diagnosis of TB and especially extra-pulmonary TB, is based on combination of tests and the main role of culture was once again confirmed in their study. ${ }^{12}$ Also, Rufai et al in their studies found that GeneXpert has low diagnostic utility for ascitic fluid, pleural fluid and cerebrospinal fluid (CSF) samples especially in India as compared to culture. ${ }^{13,14}$ In our institute, more than half of the samples sent for GeneXpert and smear were not sent for culture. One sample which was negative by Xpert but positive on smear and culture grew Mycobacterium fortuitum from lymph node which is another disadvantage of Xpert that it only detects $M T B C$ and not Atypical Mycobacteria and re-insists the importance of culture even though it is time consuming. In our case, the number of extrapulmonary samples were much higher as compared to sputum and maximum number of samples were from reproductive age group and hence culture and DST along with GeneXpert and smear should have been done in spite of WHO recommendation for this test. Hence, GeneXpert should not be used to rule out TB as it is suboptimal in ruling out TB. ${ }^{12}$ Especially in a TB hub like India, culture and DST testing along with smear, GeneXpert MTB/Rif and histopathology is the need of the time.

\section{Conclusion}

GeneXpert/MTB Rif is more sensitive than smear microscopy for detecting Mycobacterium tuberculosis complex but it should not be used as a screening test for tuberculosis. Instead, it can complement the usual detection methods.

Conflict of interest (If present, give more details): None

Study has complied with a prespecified analysis according to American association of medical college (AAMC) guidelines.

\section{Acknowledgement: None \\ Financial support: None}

\section{References}

1. Ananthnarayan R, Paniker Jayram CK. Ananthnarayan and Paniker's Textbook of Microbiology. $9^{\text {th }}$ ed. Hyderabad 500029 (A.P), India: Universities Press (India) Private Limited;2013:3-7.

2. Rakha BE, Hakeem MARA. GeneXpert MTB/Rif Assay: A Revolutionizing Method for Rapid Molecular Detection of Mycobacterium Tuberculosis in Comparison to Other Conventional methods.Int.J.Curr.Microbiol.App.Sci.2017;6(10):257380 . 
3. Denkinge MC, Schumacher GS, Boehme CC, Dendukuri N, Pai M, Steingart KR. Xpert MTB/Rif assay for the diagnosis of extrapulmonary tuberculosis: a systematic review and meta-analysis. Eur Respir J 2014;44:435-66.

4. Betty A, Sahm Daniel F, Weissfield Alice S. Bailey and Scott's Diagnostic Microbiology $.12^{\text {th }} \mathrm{ed}$. St. Louis, Missouri 63146: Mosby Elsevier:2007:478-509.

5. Loannidis P, Papaventsis D, Karabela S, Nikolaou S, Pnagi M, Raftopoulou E, et al. Cephid GeneXpert MTB/Rif assay for Mycobacterium tuberculosis detection and rifampin resistance identification in patients with substantial clinical indications of tuberculosis and smearnegative microscopy results. J Clin Microbiol 2011;49:3068-70.

6. Munir KM, Rehman S, Aasim M, Iqbal R, Saeed S. Comparison of Ziehl Neelsen Microscopy with GeneXpert for Detection of Mycobacterium Tuberculosis. IOSR-JDMS 2015;14(11):56-60.

7. Saeed M, Iram S, Hussain S, Ahmed A, Akbar M, Aslam M. GeneXpert: A new tool for the rapid detection of rifampicin resistance in mycobacterium tuberculosis $J$ Pak Med Assoc 2017;67:270-5.

8. Rufai BS, Kumar P, Singh A, Prajapati S, Balooni V, Singh S. Comparison of Xpert MTB/Rif with Line Probe Assay for Detection of Rifampin-Monoresistant Mycobacterium tuberculosis Clin Microbiol 2014;52(6):1846-52.

9. Hilleman D, Rusch-Gerdes S, Boehme C, Ritchter E. Rapid molecular detection of extrapulmonary tuberculosis by the automated GeneXpert MTB/RIF system. J. Clin. Microbiol 2011;49:1202-05.

10. Marlowe EM, Novak Weekley SM, Cumpio J, Sharp SE, Momeny MA, Babst A, Carlson JS, Kawamura M, Pandori M. Evaluation of the Cepheid Xpert MTB/RIF assay for direct detection of Mycobacterium tuberculosis complex in respiratory specimens. J. Clin. Microbiol 2011;49:1621-3.

11. Somoskovi A, Deggim V, Ciardo D, Bloemberg GV. 2013. Diagnostic implications of inconsistent results obtained with the Xpert MTB/RIF assay in detection of Mycobacterium tuberculosis isolates with an rpoB mutation associated with low-level rifampin resistance. $J$. Clin. Microbiol 2013;51:3127-9.

12. Tortoli E, Russo C, Piersimoni C, Mazzola E, Monte PD, Pascarella $\mathrm{M}$ et al. Clinical validation of Xpert MTB/Rif for the diagnosis of extrapulmonary tuberculosis. Eur Respir J 2012;40:442-7.

13. Rufai BS, Singh S, Singh A, Kumar P, Singh J, Vishal A. Performance of Xpert MTB/RIF On Ascitic Fluid Samples for Detection of Abdominal Tuberculosis. J Lab Physicians 2017;9:47-52.

14. Rufai BS, Singh A, Singh J, Kumar P, Maskar MM, Singh S. Diagnostic usefulness of Xpert mtb/rif assay for detection of tuberculosis meningitis using cerebrospinal fluid. J Infect 2017;75(2):125-13.

How to cite this article: Jangla SM, Cherian S, Naidu R. Role of GeneXpert MTB/Rif in detection of tuberculosis from clinical samples: A report from a tertiary care hospital in Mumbai. Indian J Microbiol Res 2018;5(1):143-146. 